Introduction Despite its rare ocurrence, thromboembolic events are more frequent in newborns than in any other paediatric age group, and can results of diverse congenital or acquired prothrombotic factors. We report a case of deep venous thrombosis (DVT) in a previously healthy newborn admitted with Streptococcus pyogenes infection.

Case Report 7-day old term female infant, born by vacuum delivery (birth weight $3780 \mathrm{~g}$, Apgar 9/10), referred to our NICU with a $24 \mathrm{~h}$ history of grunting, anorexia and painful, swollen and purple discoloration of left lower limb. Laboratory findings showed leucopenia (1750/uL), neutrophilia (88\%), C Reactive Protein $4.5 \mathrm{mg} / \mathrm{dL}$, normal haematocrit and platelet count. Remaining lab values, including electrolytes and coagulation tests were normal. Soft tissue infection was suspected and antibiotics started. Lower limb ultrasound and Doppler showed extensive DVT in the left side. Subcutaneous enoxaparin was started and dosage was adjusted according with anti-Xa factor levels. Homocisteyne, protein S, protein $\mathrm{C}$ and antithrombin levels were normal. Factor V Leiden, G2021A prothrombin and antiphospholipid antibodies were absent. Streptococcus pyogenes was isolated in blood and cerebrospinal fluid cultures confirming the diagnosis of sepsis and meningitis.

Discussion Thrombosis in neonatal period can cause significant morbidity and be life threatening. Early diagnosis and optimal treatment strategies are important to avoid complications. Streptococcus pyogenes has been associated with thromboembolic events in adults and older children. Despite being an uncommon cause of infection in neonates, this agent should be considered as the possible aetiology of DVT in previously healthy newborns.

\section{PO-0539 PRELIMINARY STUDY: IS GELSOLIN POSSIBLE AN EARLY DIAGNOSTIC BIOMARKER FOR NEONATAL SEPSIS?}

${ }^{1} \mathrm{H}$ Halis, ${ }^{1} \mathrm{~T}$ Gunes, ${ }^{2} \mathrm{~B}$ Saraymen, ${ }^{2} \mathrm{R}$ Saraymen. ${ }^{1}$ Pediatrics, Erciyes University Faculty of Medicine, Kayseri, Turkey; ${ }^{2}$ Biochemistry, Erciyes University Faculty of Medicine, Kayseri, Turkey

\subsection{6/archdischild-2014-307384.1183}

Background and aims Gelsolin is an actin-binding plasma protein that has a protective role against tissue injuries. Studies of sepsis are shown that diminish of plasma gelsolin (pGSN) correlates with elevated circulating levels of actin and pGSN changes correlation with clinical improvement in septic patients. The aim of this study investigate pGSN's importance in preterm infant with sepsis and related with mortality and morbidity.

Methods Thirty-one patients who were diagnosed with severe sepsis at neonatal intensive care unit were enrolled in sepsis group, twenty patients who were followed for prematurity were enrolled in control group. Plasma gelsolin levels were measured using an enzyme-linked immunosorbent assay from whole blood samples.

Result The pGSN level at the time of diagnosis in the severe sepsis group was $33.98 \pm 11.44 \mu \mathrm{g} / \mathrm{ml}$, which was significantly lower than that of 20 nonseptic preterm neonates $(60.05 \pm 11.3$ $\mu \mathrm{g} / \mathrm{ml}, \mathrm{p}<0.001)$ and after treatment $(53,38 \pm 31.26 \mu \mathrm{g} / \mathrm{ml}, \mathrm{p}$ $=0.003)$. Tollner scors in severe sepsis patients were $12.3 \pm 4$ and there were negatif correlation with gelsolin level. But, it did not detect correlation betwen postnatal age, gestational age, birth weight, gender and pGSN level in sepsis and control groups.
Conclusion Plasma gelsolin may be a usable marker for severe sepsis. Recovery of decreased gelsolin levels correlated with clinical improvement.

\section{PO-0540 CHANGES IN HAEMODYNAMIC AND PERIPHERAL PERFUSION IN SUSPECTED NEONATAL SEPSIS}

M Hassan, M Schmid, C Hanke, Marc Mendler, M Waitz, LI Huang, H Hummler. Pediatric Department, University Medical Center, UIm, Germany

\subsection{6/archdischild-2014-307384.1184}

Background Impaired hemodynamics in neonatal sepsis increase the mortality.

Objective Studying the changes in hemodynamics and peripheral perfusion in premature infants with suspected sepsis and comparing them with stable matched controls.

Methods Left (LVO) and right ventricular output (RVO), and superior vena cava (SVC) flow were measured using echocardiography in newborn infants with suspected sepsis after DOL 7. Measurements were repeated 3 times q $24 \mathrm{~h}$ and when antibiotics were discontinued. Anterior cerebral artery RI and oxygenation of the brain and the kidney were measured by near infrared spectroscopy (NIRS). Vital parameters were monitored. Controls were studied once; matched with cases for GA, BW, and postnatal age.

Results Twelve preterm infants (GA $26.8 \pm 2.5$ wks; BW $843 \pm$ $225 \mathrm{~g})$ were studied at postnatal age 13.5 (7-60) days and compared to 12 stable matched controls. Four cases had a positive blood culture, six had an elevated CrP, and 2 had an elevated IL8 only. LVO showed an increase over time reaching a maximum of $404 \pm 104 \mathrm{ml} / \mathrm{kg} / \mathrm{min}$ on the $3 \mathrm{rd}$ measurement point ( $\mathrm{p}$ $=0.046$ compared to the 1 st measurement) and was higher compared to controls $(p=0.04)$. SVC flow showed a trend towards increase over time. The control group had a higher SVC flow at the initial measurement $(p=0.05)$. No significant change in RVO, NIRS, Doppler parameters, and in blood pressure over time or compared to controls, although five cases required circulatory support either by volume therapy, catecholamines or both.

Conclusions Suspected sepsis was associated with haemodynamic changes which may have implications for clinical management.

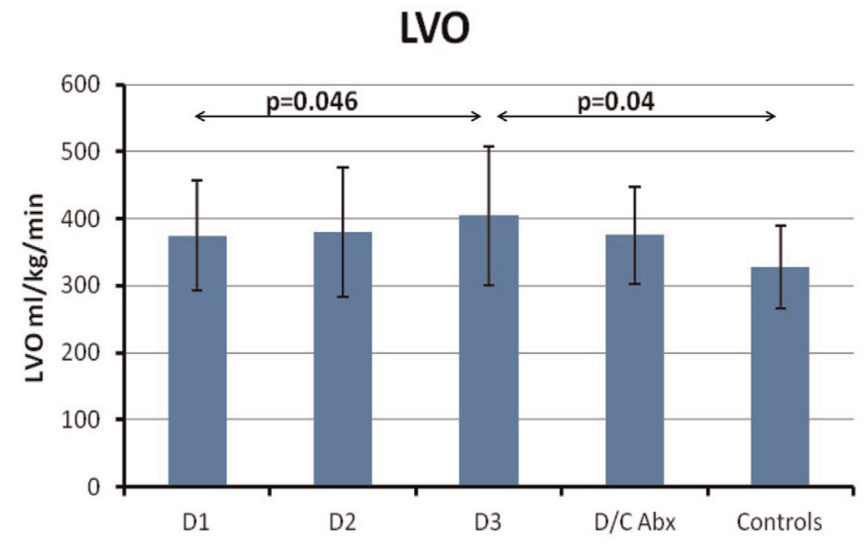

Abstract P0-0540 Figure 1 Average \pm SD of left ventricular output (LVO) changes over time, after clinical improvement and LVO in the control group. D/C Abx, discontinuation of antibiotics.

PO-0541 WITHDRAWN 